\title{
PROGRESS WITH THE SNS FRONT-END SYSTEMS*
}

\author{
R. Keller, ${ }^{* *}$ W. Abraham, J.J. Ayers, D.W. Cheng, P. Cull, R. DiGennaro, L. Doolittle, ${ }^{* *}$ R.A. Gough,** J.B.
}

Greer,** M.D. Hoff,** K.N. Leung, S. Lewis, C. Lionberger, R. MacGill, Y. Minamihara, M. Monroy, D. Oshatz,** J.

Pruyn, A. Ratti,** J. Reijonen, T. Schenkel,** J.W. Staples, ${ }^{* *}$ D. Syversrud, R. Thomae, S. Virostek,** and R. Yourd**

Ernest Orlando Lawrence Berkeley National Laboratory, Berkeley, CA

\section{Abstract}

The Front-End Systems (FES) of the Spallation Neutron Source (SNS) project have been described in detail elsewhere [1]. They comprise an rf-driven $\mathrm{H}^{-}$ion source, electrostatic LEBT, four-vane RFQ, and an elaborate MEBT. These systems are planned to be delivered to the SNS facility in Oak Ridge in June 2002. This paper discusses the latest design features, the status of development work, component fabrication and procurements, and experimental results with the first commissioned beamline elements.

\section{INTRODUCTION}

The Spallation Neutron Source (SNS) project [2] is presently in the third year of its construction phase. LBNL is building the front end (linac injector) with its main subsystems consisting of ion source, low-energy beamtransport section (LEBT), RFQ accelerator, and mediumenergy beam-transport section (MEBT). Some parts of the front end, i.e. the rf power system for the RFQ and the MEBT chopper structures with their power supplies, will be supplied by LANL; some diagnostic elements by BNL. The SNS Front-End project has been described in detail elsewhere with an ample collection of references [1], and the present paper emphasizes design changes and progress with construction. Some basic features, however, are presented here as well, to facilitate easier understanding and address recent changes in main SNS parameter values as they relate to the front end. A 3-dimensional CAD layout of the front-end beamline is shown in Figure 1, omitting all ancillary systems.

The SNS accelerator systems aim at delivering intense proton-beam pulses of less than $1-\mu$ s duration to the spallation target at $60-\mathrm{Hz}$ repetition frequency and with an average beam power of $1.44 \mathrm{MW}$. The 1-ms long $\mathrm{H}^{-}$macro pulses that are accelerated in the linac to $1-\mathrm{GeV}$ energy have to be chopped into 'mini pulses' of 645-ns duration, with 300-ns pauses.

Chopping is performed in the front end by two separate chopper systems located in LEBT and MEBT, respectively. The LEBT chopper removes most of the beam power during the mini-pulse gaps, and the MEBT chopper reduces the rise and fall time of the transported beam.

The main requirements for the SNS Front-End Systems are listed in Table 1. The front end is being assembled and commissioned at the Integrated Testing Facility at LBNL before being shipped to ORNL in the summer of 2002.

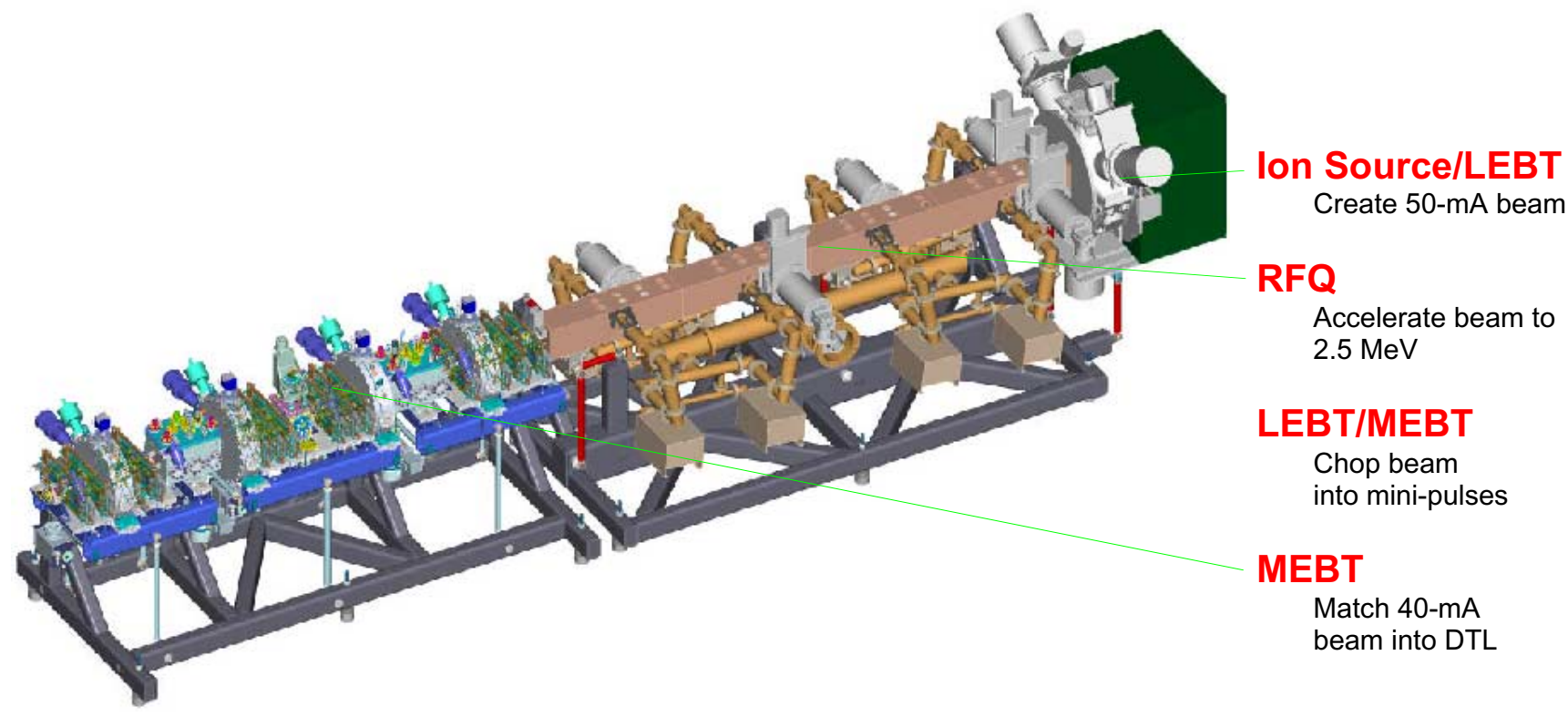

Figure 1. Layout of the SNS front-end beamline.

\footnotetext{
* Work supported by the Director, Office of Science, Office of Basic Energy Sciences, of the US Department of Energy under Contract No. DEAC03-76SF00098.

\# r_keller@lbl.gov

** Author/co-author of other contributions submitted to this conference.
} 
Table 1. FES Key Performance Parameters

\begin{tabular}{lc}
\hline Ion species & $\mathrm{H}^{-}$ \\
Output energy $(\mathrm{MeV})$ & 2.5 \\
$\mathrm{H}^{-}$peak current: & \\
$\quad$ MEBT output $(\mathrm{mA})$ & 38 \\
$\quad$ Nominal ion-source output $(\mathrm{mA})$ & 50 \\
Output normalized transverse rms emittance $(\pi \mathrm{mm} \mathrm{mrad})$ & 0.27 \\
Output normalized longitudinal rms emittance $(\pi \mathrm{MeV}$ deg $)$ & 0.126 \\
Macro pulse length (ms) & 1 \\
Duty factor $(\%)$ & 6 \\
Repetition rate (Hertz) & 60 \\
Chopper system: & \\
$\quad$ Rise, fall time (ns) & 10 \\
$\quad$ Off/on beam-current ratio & $10^{-4}$ \\
\hline
\end{tabular}

\section{ION SOURCE AND LEBT}

The $\mathrm{H}^{-}$ion source and LEBT are shown in Fig. 2. The source plasma is sustained by pulsed 2-MHz-rf power and confined by a multi-cusp magnet configuration. A magnetic dipole filter separates the main plasma from a smaller $\mathrm{H}^{-}$production region (darker area in Fig. 2) where low-energy electrons help generating copious amounts of negative ions. A heated collar, equipped with eight cesium dispensers, surrounds this $\mathrm{H}^{-}$production volume.

The outlet plate of the ion source contains another dipole-magnet configuration that creates a deflecting field across the extraction gap in order to separate extracted electrons from the ion beam and steer them towards a 'dumping' electrode biased at $5 \mathrm{kV}$ with respect to the outlet plate. Because this dumping field steers the ion beam as well, the entire plasma generator is tilted at an adjustable angle of about $3^{\circ}$ against the LEBT axis to compensate for this effect.

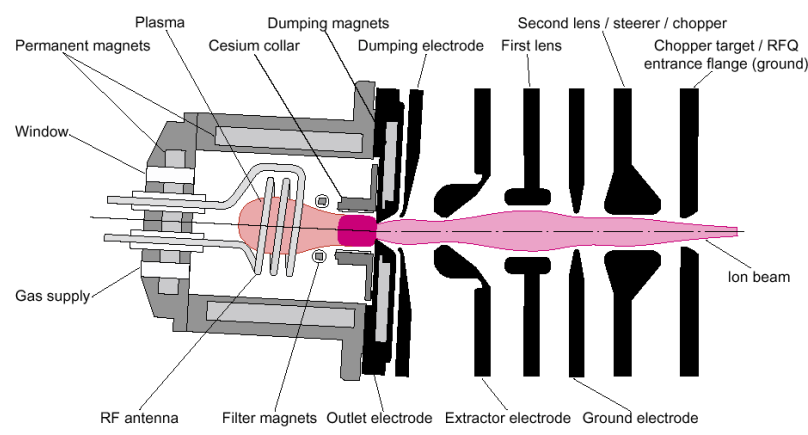

Figure 2: Schematic of the ion source and LEBT. Note that the actual filter and electron-dumping magnetic fields are oriented orthogonally to the illustration plane. The width of the ion beam is exaggerated in this schematic to emphasize the focusing action of the double-lens system.

The LEBT structure has to serve five main purposes, i.e., beam formation, 2-parameter matching into the RFQ, steering in angle and transverse offset, pre-chopping, and gas pumping. A fully electrostatic system with two einzel lenses as focusing elements was chosen for the SNS LEBT. The second one of these lenses is split into four quadrants which can be biased with d.c. and pulsed voltages to provide angular steering as well as pre-chopping. The LEBT can also be offset against the RFQ axis.
The last LEBT electrode is part of the RFQ entrance wall, and on its upstream side it carries a diagnostic element made again from four insulated quadrants. During the pauses in between mini pulses, chopping voltages of $\pm 2.5-\mathrm{kV}$ amplitude and 300-ns duration are applied to opposing pairs of lens quadrants in a rotating pattern, directing the chopped beam alternatingly towards each of the four separation zones between the diagnostic-electrode quadrants. In this way, any parts of the beam that are not intercepted by the diagnostic electrode are prevented from hitting the RFQ vane tips whose accurate shapes could otherwise gradually be eroded by sputtering.

The LEBT-electrode shapes were optimized by simulating proton beams, using the 2 -d positive-ion code IGUN [3] in a novel way that allows introduction of finite ion temperatures into the calculation without experiencing unrealistic deformations of the plasma meniscus [4]. Details of the beam-formation and electron-dumping processes were modeled [5] using the 2-d code PBGUNS [6] with actual $\mathrm{H}^{-}$ion species input and handing the trajectory data over to the 3-d code SIMION [7]. These simulations were helpful to determine the source tilt-angle and prove that no lateral axis offset was needed to obtain low emittances.

The ion source and LEBT have been commissioned, and average beam pulse-currents of $50 \mathrm{~mA}$ have been obtained at $6 \%$ duty factor and transported through the LEBT. Our simulations predict transmission values $>85 \%$ through the RFQ with the actual emittances measured by an Allison scanner and shown in Figure 3. Peak beamcurrent values up to $68 \mathrm{~mA}$ were measured at the beginning of the pulses.
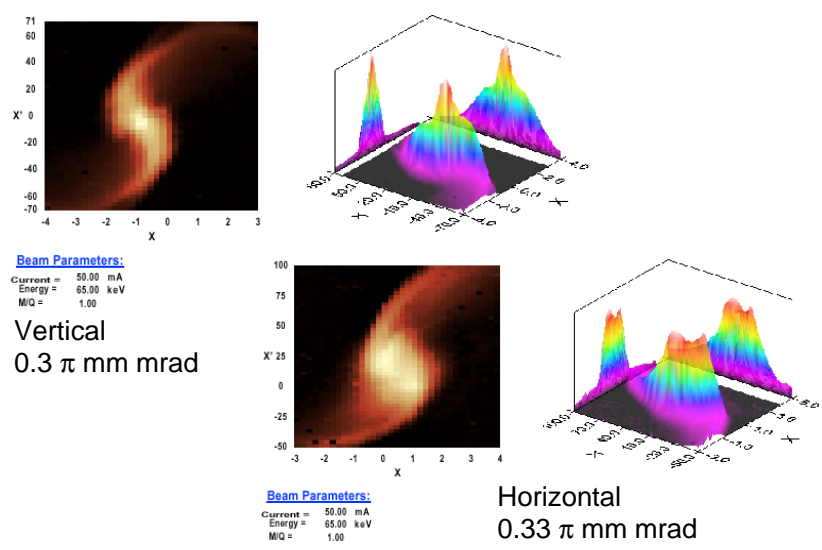

Figure 3: Transverse emittances of a 50-mA beam, taken at the LEBT exit.

The remaining issues for the ion source are the layout of the rf impedance matcher and facilitation of the discharge ignition. The most promising approach consists in using low-amplitude cw rf-power of $13.56-\mathrm{MHz}$ frequency to create a steady-state, low density plasma with negligible $\mathrm{H}^{-}$abundance, in addition to the main $2-\mathrm{MHz}$ pulsed power. Details of a newly developed matcher de- 
sign are given elsewhere [Staples**]; efforts are underway to finalize the implementation of this scheme.

\section{RFQ}

The SNS RFQ is 3.72-m long overall and consists of four modules built as composite structures with an outer GlidCop shell and four oxygen-free copper vanes. Peak surface fields reach 1.85 Kilpatrick, and the total rf power consumption is $800 \mathrm{~kW}$ during pulses. Water-cooled $\pi$ mode stabilizers separate unwanted dipole modes from the main quadrupole mode. Static frequency tuning is achieved by 20 slug tuners per module, and dynamic tuning by adjusting the temperature difference between vane tips and the outer walls of the modules.

Figure 4 shows the assembled first module before the final brazing operation. This module has now been commissioned, reaching the full $\mathrm{rf}$ gradient. The resonance frequency with slug tuners at nominal positions is very close to the design frequency of $402.5 \mathrm{MHz}$, and the field flatness is better than $\pm 1 \%$ peak-to-peak. The dynamic tuning procedure involving regulation of the vane-to-wall temperature difference was successfully tested with this module. The other three modules are all in advanced stages of fabrication; Module \#2 has been conditioned to full rf amplitude as well.

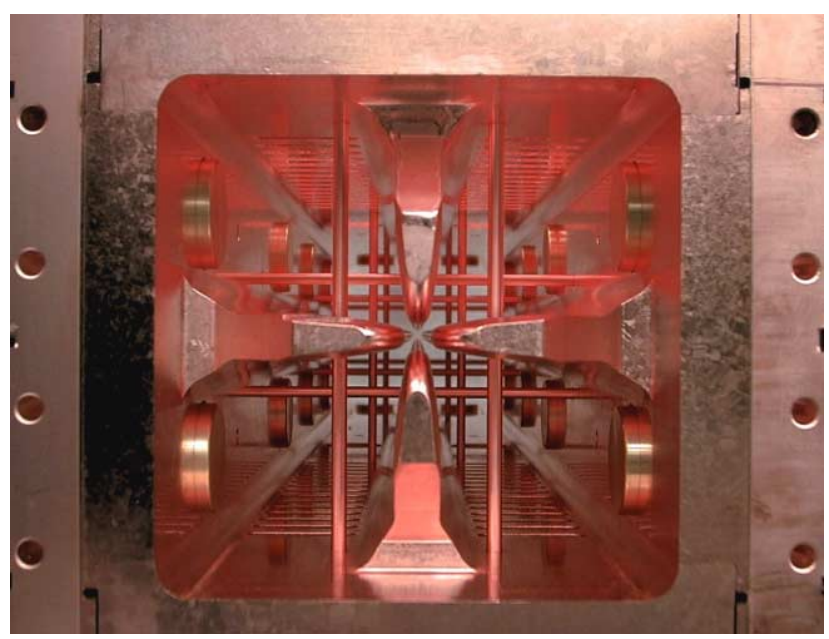

Figure 4: End-on view of the assembled RFQ Module \#1 prior to the final brazing operation. The upstream ends of the four vanes are seen at the center, with $\pi$-mode stabilizers penetrating the vanes horizontally and vertically.

The first rf-accelerated SNS beam was achieved on the first day after connecting RFQ Module \#1 to the LEBT tank. These experiments resulted in validating the structure modeling efforts and the LEBT-chopper design. As an example, Figure 5 shows the simulated and measured beam transmission values as a function of cavity excitation. The mini-pulse rise and fall times generated by the LEBT chopper amounted to $25 \mathrm{~ns}$, twice as fast as had been assumed for the design of the MEBT chopper target.

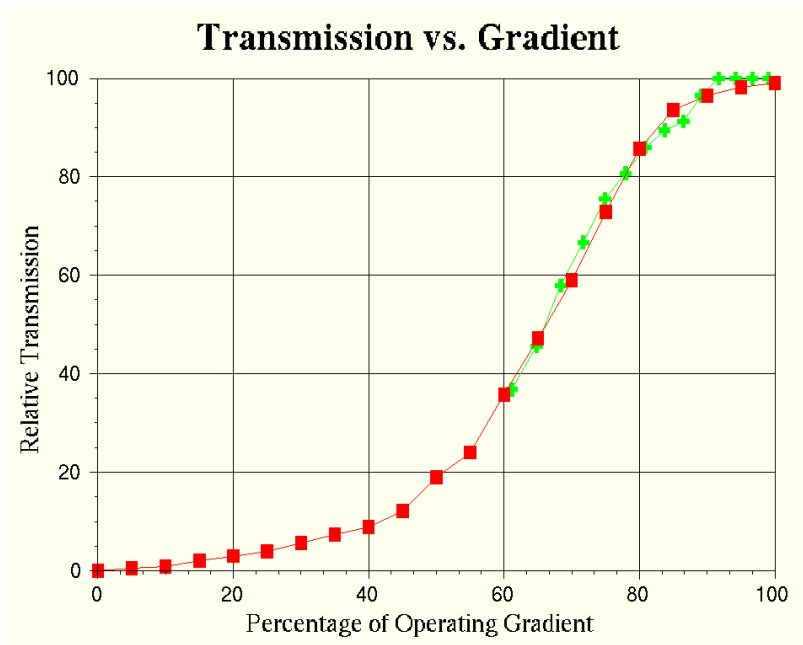

Figure 5: Simulated (red squares) and measured (green crosses) transmission values of RFQ Module \#1, normalized to $100 \%$ maximum, for an input beam of $35-\mathrm{mA}$.

\section{MEBT}

The 3.67-m long Medium-Energy Beam Transport (MEBT) structure shown in Figure 1 has three main functions, i.e., matching the beam from the RFQ exit plane into the MEBT chopper and its target, cleanup chopping, and guiding the remaining particles into the Drift-Tube Linac currently being built by LANL. Matching in both transverse and in the longitudinal direction is provided by 14 quadrupole magnets, arranged in three families, and four rebuncher cavities. An anti-chopper will direct all particles back on axis that were deflected by the chopper during the rising and falling pulse flanks and not intercepted by the target.

The MEBT will also contain diagnostic elements such as beam-position monitors that will also gather phase information, profile monitors, and two fast current transformers.

All MEBT elements are grouped on three rafts that can be individually aligned. At present, most major beamline components, including power supplies, have been fabricated and received at LBNL; power tests of the first rebuncher cavity are scheduled for June 2001.

\section{REFERENCES}

[1] R. Keller for the FES Team, "Status of the SNS Front-End Systems, Paper MOP5B04, EPAC 2000, Wien (2000).

[2] T. Mason, "The Spallation Neutron Source: A Powerful Tool for Materials Research," Paper MOAL04, these conference proceedings, PAC '01, Chicago, IL (2001)

[3] R. Becker, "New Features in the Simulation of Ion Extraction with IGUN,” EPAC '98, Stockholm (1998).

[4] J. Reijonen, R. Thomae, and R. Keller, "Evolution of the LEBT Layout for SNS," Paper MOD19, Linac 2000, Monterey (2000).

[5] R. Welton at al., "Simulation of the ion source extraction and lowenergy beam transport systems for the Spallation Neutron Source," submitted to ICIS '01, Oakland (2001).

[6] J. Boers, "PBGUNS," Thunderbird Simulations, 626 Bradfield Dr., Garland, TX 75042.

[7] D. Dahl, "SIMION 3D v.7.0," Idaho National Engineering and Environmental Laboratory Idaho Falls, ID 83415 (2000). 